\title{
Review Article \\ Review of 3D Printed Millimeter-Wave and Terahertz Passive Devices
}

\author{
Bing Zhang, ${ }^{1}$ Wei Chen, ${ }^{2}$ Yanjie Wu, ${ }^{3}$ Kang Ding, ${ }^{4}$ and Rongqiang $\mathrm{Li}^{5}$ \\ ${ }^{1}$ College of Electronics and Information Engineering, Sichuan University, Chengdu 610064, China \\ ${ }^{2}$ Department of Electrical and Computer Engineering, Faculty of Engineering, National University of Singapore, Singapore 117583 \\ ${ }^{3}$ School of Physics and Optoelectronic Engineering, Guangdong University of Technology, Guangzhou 510006, China \\ ${ }^{4}$ National Key Laboratory on Electromagnetic Environmental Effects and Electro-Optical Engineering, \\ PLA University of Science and Technology, Nanjing 210007, China \\ ${ }^{5}$ School of Electronic Engineering, Chengdu University of Information Technology, Chengdu 610225, China
}

Correspondence should be addressed to Yanjie Wu; wuyanjie@gdut.edu.cn

Received 23 March 2017; Revised 10 June 2017; Accepted 19 June 2017; Published 24 July 2017

Academic Editor: Xiulong Bao

Copyright (C) 2017 Bing Zhang et al. This is an open access article distributed under the Creative Commons Attribution License, which permits unrestricted use, distribution, and reproduction in any medium, provided the original work is properly cited.

The 3D printing technology is catching attention nowadays. It has certain advantages over the traditional fabrication processes. We give a chronical review of the $3 \mathrm{D}$ printing technology from the time it was invented. This technology has also been used to fabricate millimeter-wave (mmWave) and terahertz ( $\mathrm{THz})$ passive devices. Though promising results have been demonstrated, the challenge lies in the fabrication tolerance improvement such as dimensional tolerance and surface roughness. We propose the design methodology of high order device to circumvent the dimensional tolerance and suggest specific modelling of the surface roughness of 3D printed devices. It is believed that, with the improvement of the $3 \mathrm{D}$ printing technology and related subjects in material science and mechanical engineering, the 3D printing technology will become mainstream for mmWave and $\mathrm{THz}$ passive device fabrication.

\section{Introduction}

Terahertz (THz) waves, or submillimeter/far-infrared waves, are defined as the electromagnetic (EM) radiation in the frequency from 0.1 to $10 \mathrm{THz}$. They cover a large portion of the EM spectrum between mid-infrared and microwave bands. This spectral domain has low frequency crystalline lattice vibrations and other intermolecular vibrations in many chemical and biological materials, including explosives, drugs, and other biomolecules. Many polar gases also have the distinctive finger print in the THz spectrum. The absorbed and reflected $\mathrm{THz}$ waves of these materials contain information that is not available in other frequency ranges. Hence, the $\mathrm{THz}$ is extensively explored for sensing and imaging applications. As a potential modality for sensing and imaging, the $\mathrm{THz}$ is considered safe because of its penetration capability through obstacles like paper, textile, ceramic, wood, and leather with negligible attenuation. $\mathrm{THz}$ technologies are also used for noninvasive and nondestructive sensing of targets under covers. Recently, $\mathrm{THz}$ spectroscopy and imaging of explosive related component have been investigated for defense usage.

Compared with relatively well-developed technologies and widespread applications in microwave, mid-infrared and optical bands, research, design, and technology developments in the $\mathrm{THz}$ band are still in infancy. A $\mathrm{THz}$ system is built up of various active and passive components. For the antenna and other passive devices, their dimensions are usually proportional to the wavelength. The wavelength of the THz EM waves lies in the range of $3 \mathrm{~mm}-30 \mu \mathrm{m}$, which endows the $\mathrm{THz}$ passive devices with very small profile. A small variation of the device dimension might give rise to large shift of the functional band. Consequently, the fabrication of $\mathrm{THz}$ antenna and passive devices requires sophisticated process of tight dimensional tolerance. Moreover, the surface roughness is the other equally important 
decisive factor for the $\mathrm{THz}$ antenna and passive devices. Large surface roughness leads to increased insertion loss. Thus, the fabrication process of $\mathrm{THz}$ passive device is required to have very smooth surface finishing.

Traditional fabrication methods of $\mathrm{THz}$ passive devices are computer numerical control (CNC) machining and electrical discharge machining (EDM) $[1,2]$. The CNC process uses computer-controlled precision machine tools to cut the component out of a bulk material, in which the material utilization is low. In addition, the CNC process requires the operator to have sufficient experience and skills, which undoubtedly increases the manpower cost. The EDM etches the workpieces into the desired form by means of electrical discharge, which is usually used for machining super hard materials (such as titanium alloys, carbon steels, and cemented carbides). Its fabrication tolerance is much tighter than the CNC process, but the processing cycle is longer, and the processing cost is higher. From what is mentioned above, high labour cost, long processing cycle, and low material utilization of the traditional process are the main reasons for the high cost of $\mathrm{THz}$ passive device. The contradiction between the large demand and the unaffordable cost of $\mathrm{THz}$ devices is an urgent problem to be solved in both academia and industry.

In order to meet the requirement of high-precision machining and reduce its manufacturing costs, the 3D printing technology began to be used in $\mathrm{THz}$ device fabrication. Most of its applications are seen as the fabrication of passive devices like waveguides, horn antennas, and cavity-based components. The 3D printing technology uses powder or liquid based materials to construct the component layer by layer. Different from traditional subtractive manufacturing (SM) processes, 3D printing is an additive manufacturing (AM) process that is environmentally friendly, of low cost, of low-power consuming, and highly flexible and has a short processing cycle. It has the following advantages: (1) Efficient material utilization: raw materials that are not sintered or shaped can be used repeatedly; (2) lower energy consumption; (3) less demanding on the operator's experience and thus lower labour costs; (4) high flexibility: it is capable of realizing complex structure that is impossible to be fabricated by traditional processes, which gives designers more flexibility; (5) short turn-around time: it eliminates the tool and the mold preparation time; hence the processing cycle is shorter.

In this paper, we will investigate using the $3 \mathrm{D}$ printing technology to fabricate $\mathrm{THz}$ antennas and passive devices. Firstly, we will review the history of the 3D printing technology. Then $3 \mathrm{D}$ printed mmWave and $\mathrm{THz}$ passive devices will be introduced. Thirdly, we will elaborate on the key factors for the performance of $3 \mathrm{D}$ printed mmWave and $\mathrm{THz}$ passive devices which are surface roughness and dimensional tolerance. Finally we will conclude the paper with prospects on the development trend of using the 3D printing technology for $\mathrm{mmW}$ ave and $\mathrm{THz}$ passive device fabrication.

\section{History of the 3D Printing Technology}

Generally, the 3D printing technologies could be categorized as binding and depositing in terms of processes and metallic and dielectric in terms of materials. Figure 1 shows the history of 3D printing technology since it was invented in 1980, when Kodama applied a patent on rapid prototyping (RP). In 1986, a patent on stereolithography apparatus (SLA) was issued to Hull [3]. In 1992, Deckard et al. filed a patent on selective laser sintering [4]. Crump filed a patent on fused deposition modelling (FDM) in 1992 [5]. ARCAM was established in 1997 to develop the electron beam melting (EBM) technology [6]. MCP Technologies introduced the selective laser melting (SLM) in 2000 [7].

\section{Review of 3D Printed Millimeter-Wave and Terahertz Passive Devices}

3D printed mmWave and $\mathrm{THz}$ devices fall into categories of passive and active devices considering the functionalities. Because of the dimensional tolerance of existing 3D printing technologies, limited by the required gate length of mmWave transistors, it is very challenging to print a transistor of $f_{T}$ and $f_{\max }$ falling in the mmWave spectrum. Most of the 3D printed mmWave and $\mathrm{THz}$ passive devices are seen as lenses, electromagnetic bandgap (EBG) structures, waveguide filters, and horns.

The first 3D printed mmWave passive device is a dual polarized horn reported in 2005 [8]. It was printed by SLA using Emerson and Coming $\mathrm{HiK}$ dielectric powder. It has an averaged gain of $12 \mathrm{dBi}$ over the $34-40 \mathrm{GHz}$ bandwidth. A Ka-band Luneburg lens with average $23 \mathrm{dBi}$ gain was printed by ceramic stereolithography apparatus (CSLA) on photoreactive alumina in 2007 [9]. The CSLA was also used to print a Ka-band EBG bandpass filter (BPF) [10]. The filter has the center frequency of $32.94 \mathrm{GHz}$ with $1.03 \%$ bandwidth. Frequency shift is caused by the dimensional tolerance in printing and shrinkage in postsintering. In 2008, a D-band (110-170 GHz) EBG resonator was printed by the micro SLA ( $\mu$ SLA) technology on alumina [11]. This work pushed the boundary of $3 \mathrm{D}$ printed devices from mmWave to $\mathrm{THz}$ spectrum. Lee et al. used the CSLA to print a Wband (75-110 GHz) EBG structure [12]. It has the $84-118 \mathrm{GHz}$ bandgap. Then they transformed the EBG into an aperture antenna of $25 \mathrm{dBi}$ gain [13]. Wu et al. conducted a series of experiments on the polymer jetting (PJ) technology. They firstly reported a $600 \mathrm{GHz}$ woodpile structure (WPS) EBG and a $350 \mathrm{GHz}$ Johnson EBG in 2008 [14], a G-band (140-220 GHz) hollow-core electromagnetic crystal (EXMT) waveguide in 2011 [15], and a D-band hollow-core EXMT antenna of $25 \mathrm{dBi}$ gain in 2012 [16]. A Q-band $(33-50 \mathrm{GHz})$ Luneburg lens was printed by Nguyen in 2010 [17], with 55-65 GHz bandwidth and $21 \mathrm{dBi}$ gain. A W-band corrugated horn and waveguide were printed by SLA using UV-polymer in 2011 [18]. They were printed in split pieces and metal plated in postprocess. In 2013, a polymer jetted acrylic resin $\mathrm{THz}$ waveguide was reported [19]. A polymer jetted W-band dielectric reflectarray was introduced by Nayeri et al. in 2014 [20]. Later, in 2014, an H-band (220-325 GHz) waveguide and diagonal horn by SLA showed up [21]. Qu et al. reported an H-band SLA lens of $26.5 \mathrm{dBi}$ gain [22]. In 2015, a Kaband $(26.5-40 \mathrm{GHz})$ offset stepped-reflector antenna was 


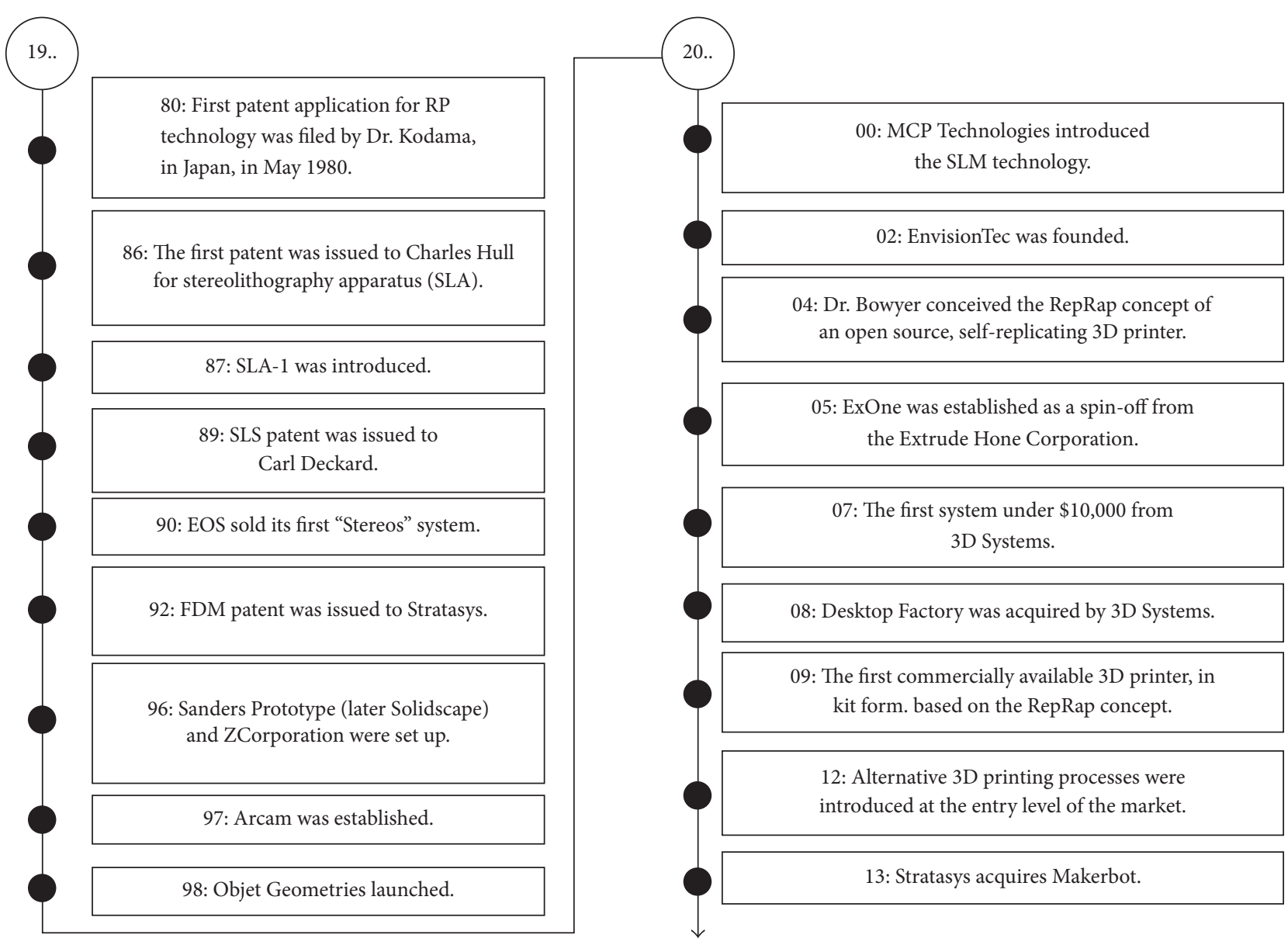

FIGURE 1: Chronicle of the 3D printing technology.

reported using SLS sintered nylon-polyamide [23]. A Wband waveguide and a W-band 6-order BPF were printed by D'Auria et al. in 2015 [24]. Besides, there are also works reported using $3 \mathrm{D}$ printing technologies for low frequency passive device fabrications [25-38].

From what is mentioned above, it can be seen that for most of the dielectric 3D printed passive devices the fabrication process is to first print the device in split pieces and then to metal plate and finally to assemble. Compared with the traditional CNC and EDM metallic devices, they have advantages of light weight and low cost. However, the disadvantages are quite obvious: (1) The process of metal plating and assembly increased the process complexity. (2) The low mechanical strength of plastic and resin affects the life of the device. (3) Pores in the dielectric body and different coefficient of thermal expansion make it impossible to be used in harsh environments such as space. (4) The dielectric body ESD phenomenon will affect the other devices in the system. Metallic 3D printed devices effectively solve the problems due to the following: (1) Good metal conductivity: metallic 3D printed $\mathrm{THz}$ devices can be printed in one run and do not require gold plating, which greatly reduces the process complexity and manufacturing costs. (2) The physical strength of the metal makes the device life significantly extended. (3) There is no thermal expansion related issue since the metal plating is not needed. (4) The ESD problem is minor in metallic device.

Before 2012, the only reported metallic 3D printed mmWave device was an SLM $50 \mathrm{~mm}$ waveguide [39]. In 2015, Zhang from Chalmers University of Technology conducted a series of experiments on metallic 3D printed mmWave and $\mathrm{THz}$ devices [40]. Firstly, six V-band (50-75 GHz) horn antennas were printed to select the appropriate "material + process + postprocess" for our purpose. Processes used are SLM and binder jetting. Materials are $\mathrm{Cu}-15 \mathrm{Sn}$ and 316L stainless steel. Postprocesses adopted to improve the surface roughness are manual polishing, gold plating, and the micromachined process (MMP). Figure 2 shows the surface profile of the 3D printed V-band horns using ZeGage optical surface profilometer. Table 1 compares the measured surface roughness. The SLM Cu-15Sn with MMP rendered the most satisfactory surface roughness of $\mathrm{Sa}=0.25 \mu \mathrm{m}$ for mmWave and $\mathrm{THz}$ applications. However, the cost of MMP treatment equals the SLM printing cost. Balancing between the cost and performance, the SLM Cu-15Sn with manual polishing was chosen as the formula for following experiments [41].

A series of mmWave and $\mathrm{THz}$ devices were printed after the formula of "material + process + postprocess" is 


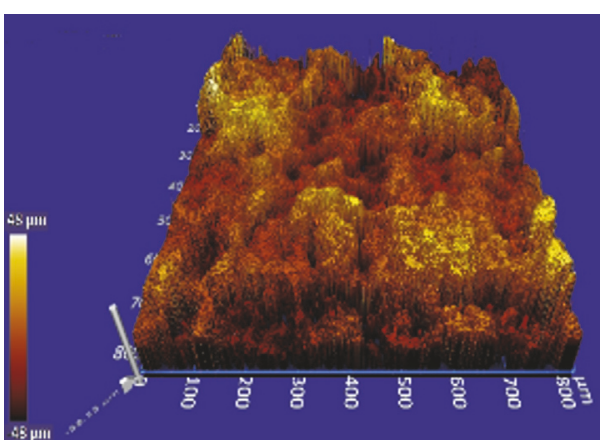

(a)

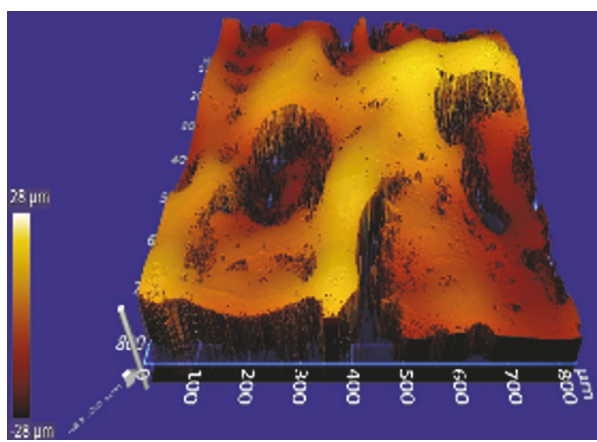

(c)

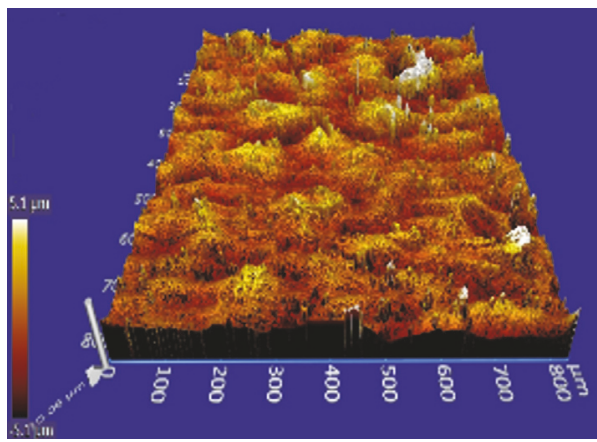

(e)

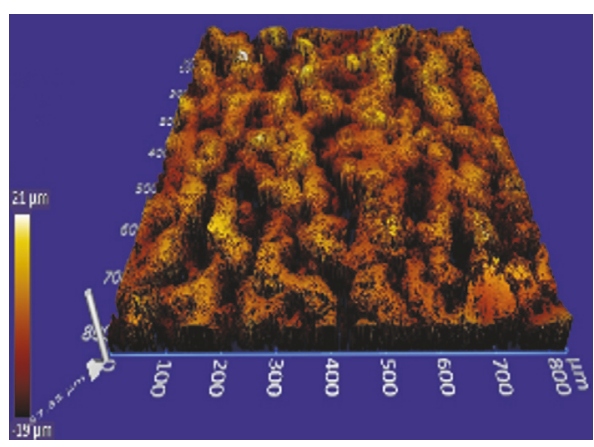

(b)

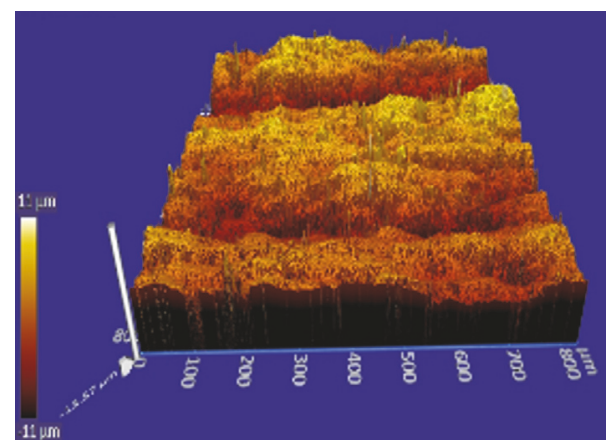

(d)

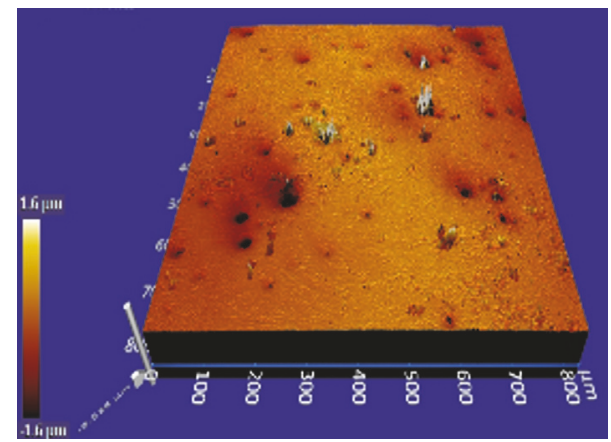

(f)

Figure 2: Roughness of 3D printed V-band horns' inner surfaces: (a) binder jetted 316L stainless steel without treatment, (b) gold electroplated binder jetted 316L stainless steel, (c) MMP treated binder jetted 316L stainless steel, (d) manually polished SLM Cu-15Sn, (e) gold electroplated SLM Cu-15Sn, and (f) MMP treated SLM Cu-15Sn.

TABLE 1: Measured inner surface roughness of V-band horns.

\begin{tabular}{lcccc}
\hline Sample & Spv $(\mu \mathrm{m})$ & Sq $(\mu \mathrm{m})$ & Sa $(\mu \mathrm{m})$ & IsoFlatness $(\mu \mathrm{m})$ \\
\hline (a) & 129.00 & 16.10 & 12.89 & 127.42 \\
(b) & 142.84 & 6.73 & 4.64 & 128.27 \\
(c) & 67.74 & 9.46 & 7.82 & 59.56 \\
(d) & 30.10 & 3.52 & 2.79 & 25.49 \\
(e) & 20.50 & 1.67 & 1.29 & 19.89 \\
(f) & 39.35 & 0.54 & 0.25 & 37.67 \\
\hline
\end{tabular}

chosen. E- (60-90 GHz), D- (110-170 GHz), and H-band $(220-325 \mathrm{GHz}) 3 \mathrm{D}$ printed horn antennas were reported to be of comparable performance with commercial counterparts, while featuring considerable cost reduction in Figure 3 [42]. Because most of the horn's surface is touchable by the polishing kit, the 3D printed horn antennas feature very good surface finishing of $\mathrm{Sa}=3 \mu \mathrm{m}$. Waveguides are also printed at the E-(WR-12), D-(WR-06), and H-band (WR-03) in Figure 4 [43]. $\pm 5 \%$ dimensional tolerance is observed. Since the cross section of the waveguide is in the scale of millimeter, 


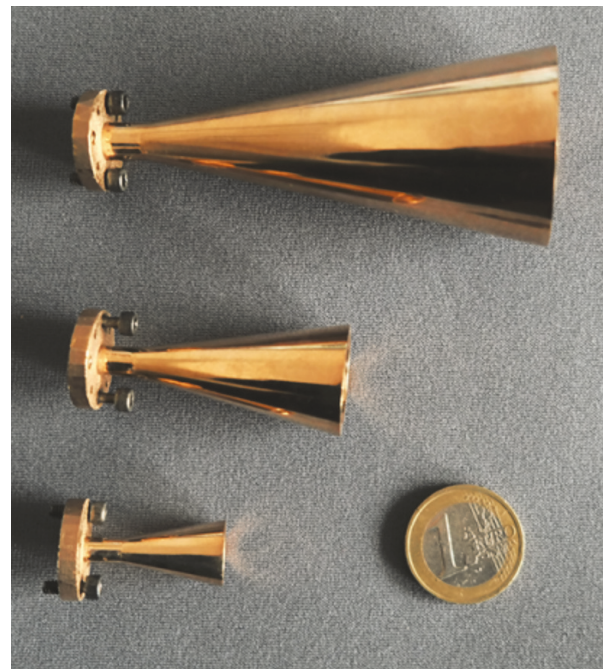

FIGURE 3: 3D printed horns antennas, from top E-, D-, and H-band.

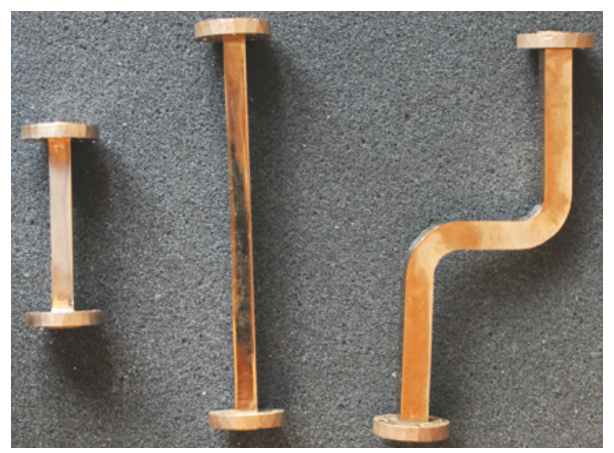

FiguRE 4: 3D printed waveguides, from left: $50 \mathrm{~mm}$ straight waveguide, $100 \mathrm{~mm}$ straight waveguide, and bend waveguide.

barely anything could be done to improve the inner surface roughness. As a result, the skin depth related insertion loss brought about by the surface roughness becomes apparent when the functional frequency increases. In general, the 3D printed waveguides show acceptable performance up to the E-band. For the D- and $\mathrm{H}$-band 3D printed waveguide, space remains for the improvement of dimensional tolerance and surface roughness. Attempts have been made to print Eband BPF in Figure 5 [44]. Two Chebyshev waveguide iris BPFs of passbands $71-76 \mathrm{GHz}$ and $81-86 \mathrm{GHz}$ were printed. Subject to the undesirable dimensional tolerance and surface roughness, the BPFs demonstrate promising behavioral agreement between simulation and measurement. The $3 \mathrm{D}$ printed BPFs are assembled as a diplexer (Figure 6) and then formed together with the 3D printed E-band horn into a front end (Figure 7) [45]. Though behavioral agreement is observed between the simulation and measurement, the accumulated influence of the dimensional tolerance and surface roughness from different component of the front end becomes obvious from the shifted functional band and deteriorated insertion loss.

\section{Challenges and Solutions for 3D Printed MmWave and THz Devices}

The two dominantly influential factors on the performance of 3D printed mmWave and $\mathrm{THz}$ devices are and dimensional tolerance and surface roughness. The former is decided by the material particle size, thermal shrinkage in sintering and postsintering, laser/electron beam size, and movement control of the laser/electron beam and the nozzle size. The latter is of more concern in $3 \mathrm{D}$ printing. It is related to material particle size, laser/electron beam size, density of the material powder, the Gaussianity of the laser beam, and the movement control of the laser/electron beam. Existing techniques to improve the surface quality of $3 \mathrm{D}$ printed devices include mechanical polishing, chemical polishing, MMP, and electroplating. However, limited by the existing process, effective improvement of the dimensional tolerance and surface roughness is difficult. Methodology to circumvent the dimensional tolerance would lead the 3D printed mmWave and $\mathrm{THz}$ device to a great leap forward to widespread applications. Accurate model to predict and 


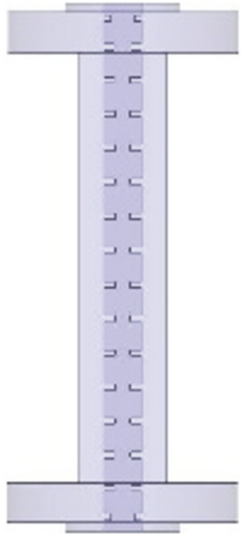

(a)

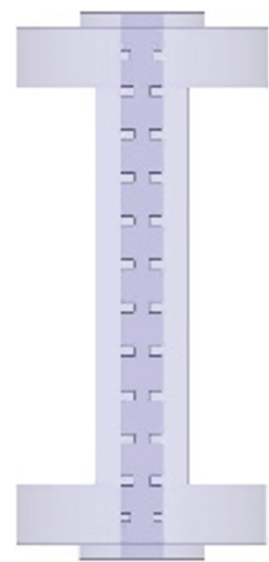

(d)

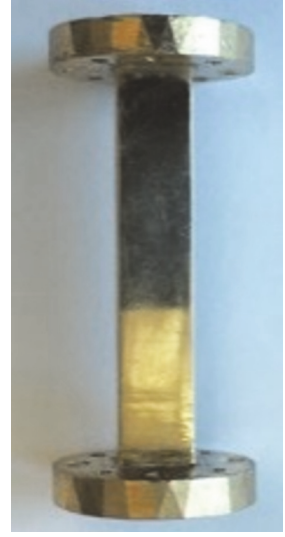

(b)

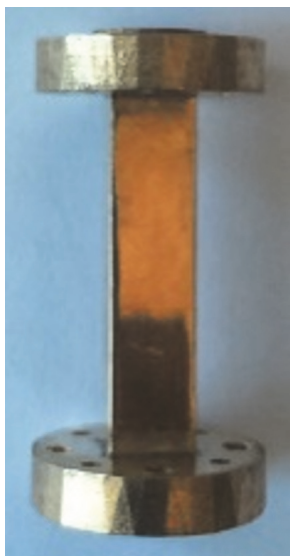

(e)

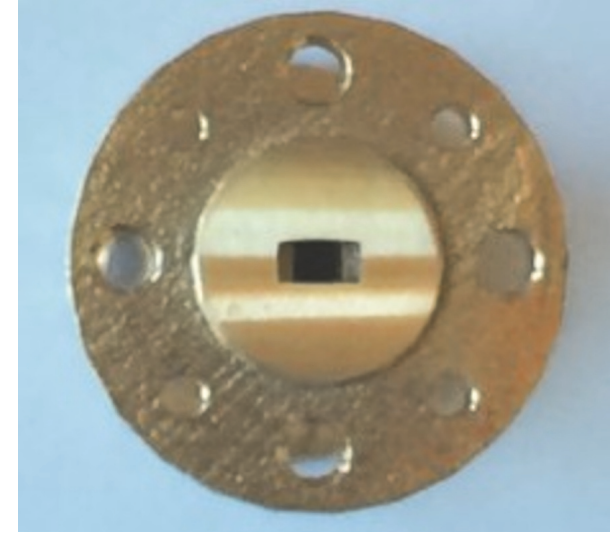

(c)

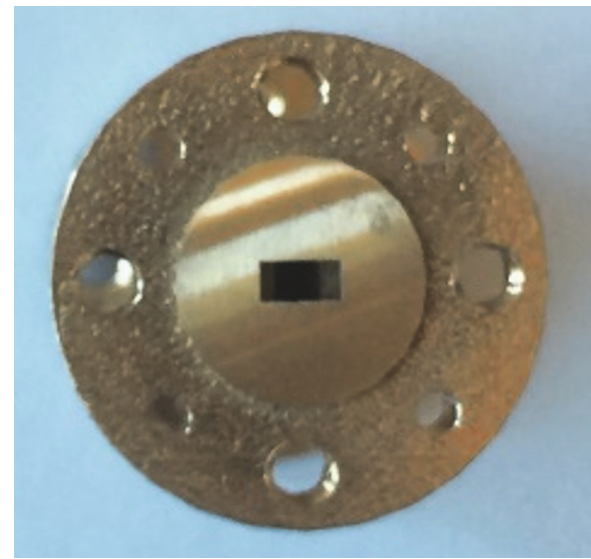

(f)

FIGURE 5: 3D printed BPFs: (a) 71-76 GHz BPF perspective view, (b) photograph, and (c) flange view; (d) 81-86 GHz BPF perspective view, (e) photograph, and (f) flange view.

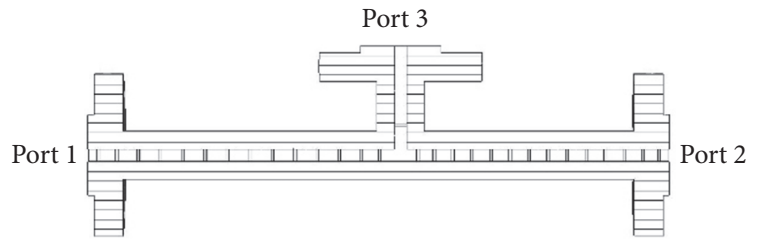

(a)

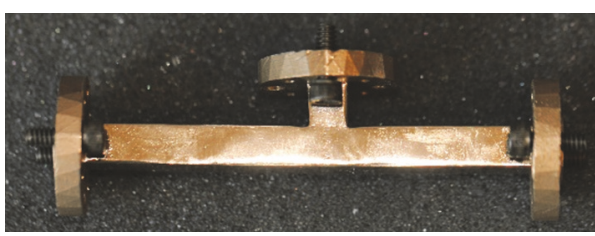

(b)

Figure 6: 3D printed E-band diplexer: (a) perspective view and (b) photograph.

evaluate the surface roughness related loss in $3 \mathrm{D}$ printed devices is valuable for quality control in industry.

4.1. Dimensional Tolerance. The influence of dimensional tolerance is reflected by deteriorated reflection coefficient, increased insertion loss, and severe passive intermodulation. It is more meaningful to avoid the dimensional tolerance under the premise that the improvement of dimensional tolerance of $3 \mathrm{D}$ printing technologies has come to a limit. The high order mode structure expands the volume of fundamental mode structure, thereby weakening the influence of the processing volume tolerance on the device performance.
However, by doing this high order modes are excited. For example, as shown in Figure 8, after transforming a fundamental mode V-band $(60-90 \mathrm{GHz})$ waveguide twist into a high order mode waveguide twist, its immunity to the dimensional tolerance is significantly improved at the cost of high order mode excitation. The flatness of the insertion loss of high order mode waveguides appears to deteriorate. The performance of the high order mode waveguides is still comparable to that of the standard fundamental mode waveguides in a narrow band (e.g., $66-74 \mathrm{GHz}$ ). In practice, most RF passive components are not required to function in a full bandwidth. The high order design methodology 


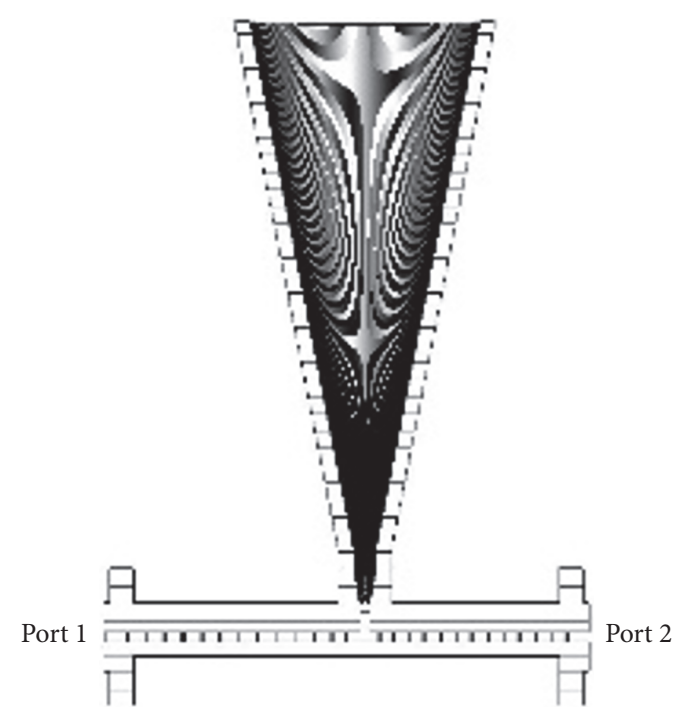

(a)

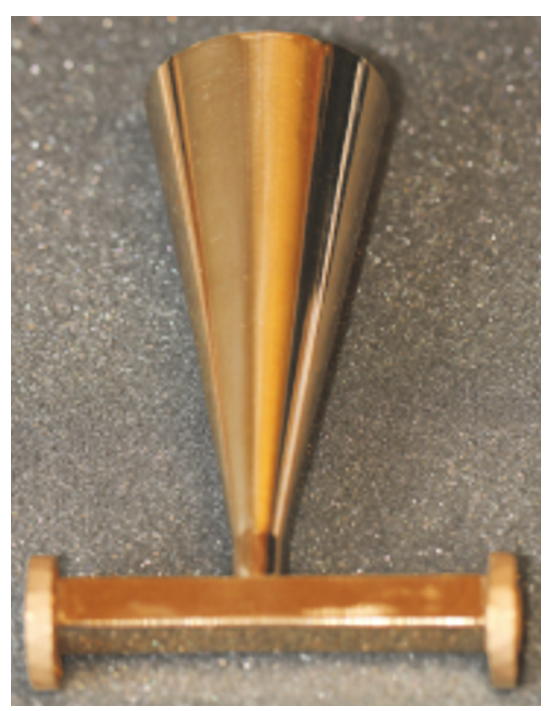

(b)

Figure 7: 3D printed E-band front end: (a) perspective view and (b) photograph.

can effectively avoid the effect of dimensional tolerance on the device performance while guaranteeing the required functionality in a specific bandwidth.

4.2. Surface Roughness. Not-well-controlled surface roughness may give rise to increased insertion loss and deteriorated passive intermodulation (PIM). Hammerstad and Jensen model, Huray model, and Hall models are representative classic roughness empirical models. They model the rough surface as a two-dimensional structure with periodic properties. These models have reference value in the analysis of conductor loss in planar transmission line structure. Depending on the material and process, different correction factors are introduced for specific applications, such as the correction factor $K$ introduced in the Hammerstad and Jensen model:

$$
K=1+\frac{2}{\pi} \tan ^{-1}\left(1.4 \frac{R_{q}}{\delta}\right) .
$$

However, the above models are only applicable to the analysis of roughness related losses in planar microstrip structures; there is no effective model for the roughness analysis of $3 \mathrm{D}$ printed $\mathrm{THz}$ devices. The $3 \mathrm{D}$ printed device is different from the traditional device in the roughness characterization due to the specific process and material. For example, the $3 \mathrm{D}$ printed surface roughness is limited by the material particle diameter; the same printer using different materials may result in obviously different surface finishing. Due to the nonuniform particle diameter of the material, the root mean square of surface roughness is usually larger than that of the conventional device in roughness. Since the device is printed layerwise and the laser used usually features a Gaussian beam, the roughness of the metal 3D printing device may be subject to periodic distribution in large scale and Gaussian distribution in small scale. Based on the classic model, a correction factor of the $3 \mathrm{D}$ printed $\mathrm{THz}$ device should be introduced, and the empirical formula of the device loss and roughness should be established. The establishment of the statistic model of the 3D printed surface roughness will benefit the academia in device performance prediction and the industry in quality control.

\section{Conclusions}

This paper reviews the state-of-the-art 3D printed mmWave and $\mathrm{THz}$ devices. They largely fall into the dielectric and the metallic categories. The dielectric 3D printed devices stand out with low body mass, while the thermal stability, physical stability, and process complexity are of concern. The metallic 3D printed devices outperform in the thermal stability and physical stability at the cost of increased body loading. The widespread applications of using 3D printing technology for mmWave and $\mathrm{THz}$ device fabrication had come to a bottleneck because of the limited dimensional tolerance and surface roughness. By adopting the methodology of high order device, the dimensional tolerance could be circumvented. A precise model of $3 \mathrm{D}$ printed surface roughness could be helpful to predict and evaluate the roughness related loss. Besides, a hybrid printing technology that merges with the traditional CNC process is recently available. The printing and machining processes are carried out within a single machine at intervals. This effectively improves the dimensional tolerance and surface roughness by the machining process, while remaining eco-friendly and cost-effective by the $3 \mathrm{D}$ printing technology. A quasi $3 \mathrm{D}$ printing process, or $2.5 \mathrm{D}$ printing process, is under development. It merges with mask printing for dimensional tolerance control. It is very promising that, with the development of $3 \mathrm{D}$ printing technology and disciplines in material science and mechanical engineering, 3D printed mmWave and $\mathrm{THz}$ 


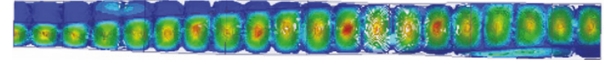

(a)

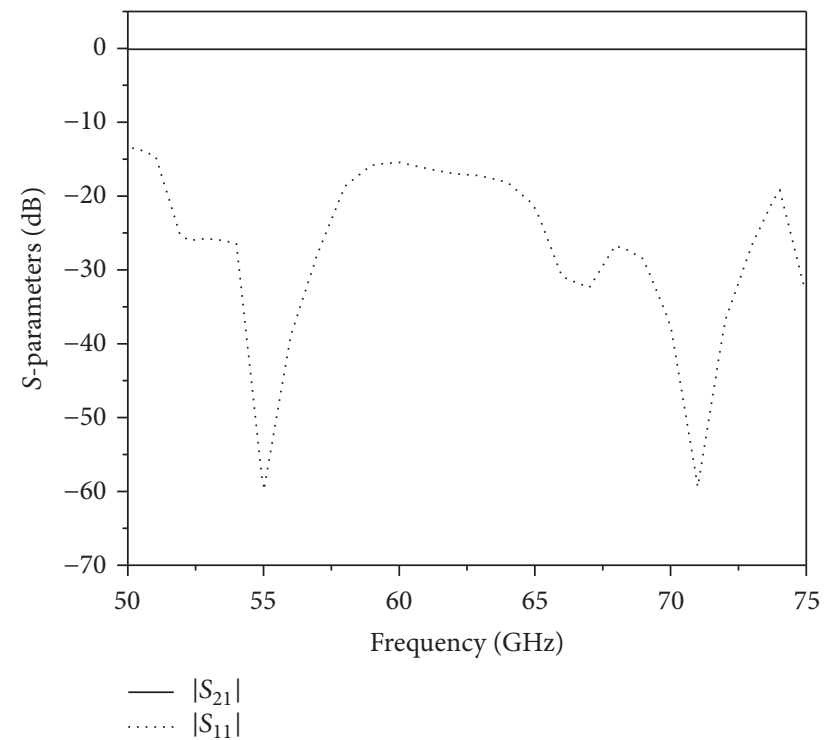

(b)

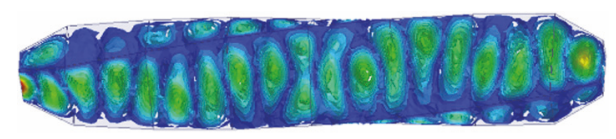

(c)

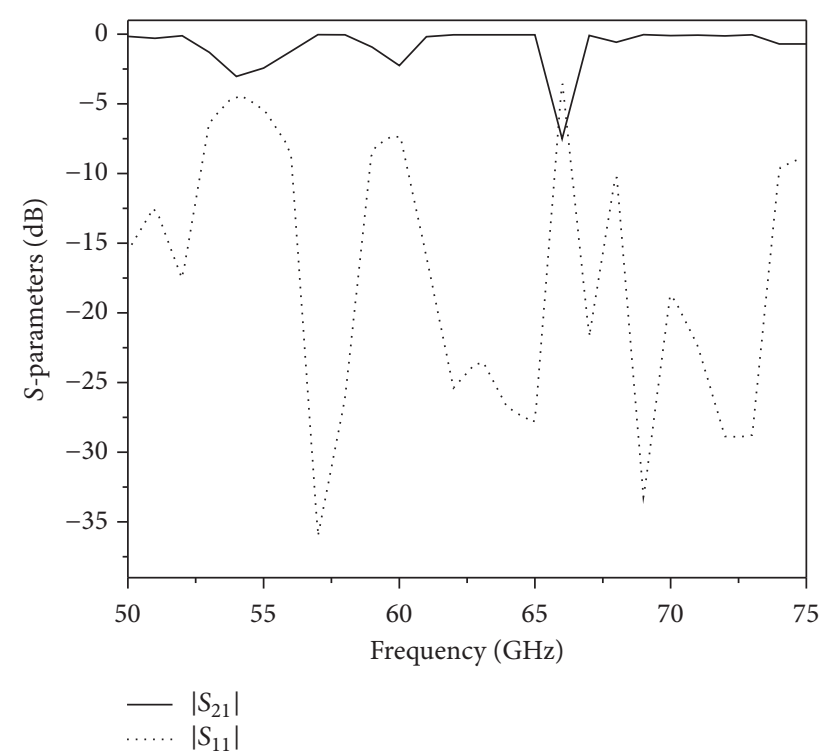

(d)

FIGURE 8: Fundamental mode and high order mode waveguide twists: (a) V-band fundamental mode waveguide twist, (b) frequency response of the $\mathrm{V}$-band fundamental mode waveguide twist, (c) V-band high order mode waveguide twist, and (d) frequency response of the V-band high order mode waveguide twist.

device will become the mainstream solution in both academia and industry.

\section{Conflicts of Interest}

The authors declare there are no conflicts of interest regarding the publication of this paper.

\section{Acknowledgments}

This work was supported by the Startup Foundation for Young Talent from Sichuan University, Research Project of Guangdong Province (2013B090500035), Youth Foundation of Guangdong University of Technology (15ZK0038), and Science and Technology Program of Guangzhou (2014J4100202). This work was also supported by the Sichuan Provincial Science and Technology Project of China (2015FZ0112), the Foundation of Chengdu University of Information Technology (J201602), and the Scholarship from China Scholarship Council (201508515023).

\section{References}

[1] X. Shang, M. Ke, Y. Wang, and M. J. Lancaster, "WR-3 band waveguides and filters fabricated using SU8 photoresist micromachining technology," IEEE Transactions on Terahertz Science and Technology, vol. 2, no. 6, pp. 477-448, 2012.

[2] F. Caspers and E. Neumann, "Optical-fiber end preparation by spark erosion," Electronics Letters, vol. 12, no. 17, 1976.

[3] C. Hull, Apparatus for production of three-dimensional object by stereolithography, U.S. Patent 4575 330, 1986.

[4] C. Deckard, J. Beaman, and J. Darrah, Method for selective laser sintering with layerwise cross-scanning, U.S. Patent 5155 324, 1992.

[5] S. Crump, Apparatus and method for creating threedimensional objects, U.S. Patent 5 121 329, 1992.

[6] ARCAM, Electron Beam Melting, 2016, http://www.arcam.com/ technology/electron-beam-melting/.

[7] SLM Solutions, SLM, 2016, http://slm-solutions.com/about-slm.

[8] L. Schulwitz and A. Mortazawi, "A compact dual-polarized multibeam phased-array architecture for millimeter-wave radar," IEEE Transactions on Microwave Theory and Techniques, vol. 53, no. 11, pp. 3588-3594, 2005.

[9] K. F. Brakora, J. Halloran, and K. Sarabandi, "Design of 3-D monolithic MMW antennas using ceramic stereolithography," IEEE Transactions on Antennas and Propagation, vol. 55, no. 3, pp. 790-797, 2007.

[10] N. Delhote, D. Baillargeat, S. Verdeyme, C. Delage, and C. Chaput, "Ceramic layer-by-layer stereolithography for the manufacturing of 3-D millimeter-wave filters," IEEE Transactions on Microwave Theory and Techniques, vol. 55, no. 3, pp. 548-554, 2007.

[11] T. Chartier, C. Duterte, N. Delhote et al., "Fabrication of millimeter wave components via ceramic stereo- and microstereolithography processes," Journal of the American Ceramic Society, vol. 91, no. 8, pp. 2469-2474, 2008.

[12] Y. Lee, X. Lu, Y. Hao et al., "Rapid prototyping of ceramic millimeterwave metamaterials: simulations and experiments," Microwave and Optical Technology Letters, vol. 49, no. 9, pp. 2090-2093, 2007.

[13] Y. Lee, X. Lu, Y. Hao, S. Yang, J. R. G. Evans, and C. G. Parini, "Directive millimetrewave antennas using freeformed ceramic metamaterials in planar and cylindrical forms," in Proceedings 
of the 2008 IEEE International Symposium on Antennas and Propagation and USNC/URSI National Radio Science Meeting, APSURSI, 4, 1 pages, San Diego, Calf, USA, July 2008.

[14] Z. Wu, J. Kinast, M. E. Gehm, and H. Xin, "Rapid and inexpensive fabrication of terahertz electromagnetic bandgap structures," Optics Express, vol. 16, no. 21, pp. 16442-16451, 2008.

[15] Z. Wu, W.-R. Ng, M. E. Gehm, and H. Xin, “Terahertz electromagnetic crystal waveguide fabricated by polymer jetting rapid prototyping," Optics Express, vol. 19, no. 5, pp. 3962-3972, 2011.

[16] Z. Wu, M. Liang, W.-R. Ng, M. Gehm, and H. Xin, "Terahertz horn antenna based on hollow-core electromagnetic crystal (EMXT) structure," IEEE Transactions on Antennas and Propagation, vol. 60, no. 12, pp. 5557-5563, 2012.

[17] N. T. Nguyen, N. Delhote, M. Ettorre, D. Baillargeat, L. Le Coq, and R. Sauleau, "Design and characterization of $60-\mathrm{GHz}$ integrated lens antennas fabricated through ceramic stereolithography," IEEE Transactions on Antennas and Propagation, vol. 58, no. 8, pp. 2757-2762, 2010.

[18] P. T. Timbie, J. Grade, D. Van Der Weide, B. Maffei, and G. Pisano, "Stereolithographed MM-wave corrugated horn antennas," in Proceedings of the 36th International Conference on Infrared, Millimeter, and Terahertz Waves (IRMMW-THz '11), pp. 1-3, Houston, Tex, USA, October 2011.

[19] S. Pandey, B. Gupta, and A. Nahata, "Terahertz plasmonic waveguides created via 3D printing," Optics Express, vol. 21, no. 21, pp. 24422-24430, 2013.

[20] P. Nayeri, M. Liang, R. A. Sabory-Garcia et al., “3D printed dielectric reflectarrays: low-cost high-gain antennas at sub-millimeter waves," IEEE Transactions on Antennas and Propagation, vol. 62, no. 4, pp. 2000-2008, 2014.

[21] A. Von Bieren, E. De Rijk, J.-P. Ansermet, and A. Macor, "Monolithic metal-coated plastic components for mm-wave applications," in Proceedings of the 39th International Conference on Infrared, Millimeter and Terahertz Waves (IRMMW-THz'14), 2, 1 pages, Tucson, Ariz, USA, September 2014.

[22] S. Qu, H. Yi, C. H. Chan, and K. B. Ng, "Low-cost discrete dielectric terahertz lens antenna using 3D printing," in Proceedings of the 2014 IEEE Conference on Antenna Measurements \& Applications (CAMA), pp. 1-3, Antibes Juan-les-Pins, France, November 2014.

[23] L. Menéndez, O. Kim, F. Persson, M. Nielson, and O. Breinbjerg, "3D printed 20/30-GHz dual-band offset stepped-reflector antenna," in Proceedings of the 9th European Conference on Antennas and Propagation (EuCAP '15), pp. 1-2, Lisbon, Portugal, May 2015.

[24] M. D’Auria, W. J. Otter, J. Hazell et al., "3-D printed metal-pipe rectangular waveguides," IEEE Transactions on Components, Packaging and Manufacturing Technology, vol. 5, no. 9, pp. 13391349, 2015.

[25] C. Guo, X. Shang, J. Li, F. Zhang, M. J. Lancaster, and J. Xu, "A lightweight 3-D printed X-band bandpass filter based on spherical dual-mode resonators," IEEE Microwave and Wireless Components Letters, vol. 26, no. 8, pp. 568-570, 2016.

[26] W. J. Otter and S. Lucyszyn, "3-D printing of microwave components for 21st century applications," in Proceedings of the 2016 IEEE MTT-S International Microwave Workshop Series on Advanced Materials and Processes for $\mathrm{RF}$ and $\mathrm{THz}$ Applications (IMWS-AMP '16), Chengdu, China, July 2016.

[27] L. Araujo, X. Shang, M. Lancaster et al., "3-D printed band-pass combline filter," Microwave and Optical Technology Letters, vol. 59, no. 6, pp. 1388-1390, 2017.
[28] C. Guo, J. Li, D. D. Dinh, X. Shang, M. J. Lancaster, and J. $\mathrm{Xu}$, "Ceramic filled resin based 3D printed X-band dual-mode bandpass filter with enhanced thermal handling capability," Electronics Letters, vol. 52, no. 23, pp. 1929-1931, 2016.

[29] C. Guo, X. Shang, J. Li, M. J. Lancaster, and J. Xu, “3-D printed lightweight microwave waveguide devices," in Proceedings of the 2016 IEEE 5th Asia-Pacific Conference on Antennas and Propagation (APCAP), Kaohsiung, Taiwan, July 2016.

[30] P. Booth and E. V. Lluch, "Enhancing the performance of waveguide filters using additive manufacturing," Proceedings of the IEEE, vol. 105, no. 4, pp. 613-619, 2017.

[31] W. J. Otter and S. Lucyszyn, "Hybrid 3-D printing technology for tunable THz applications," Proceedings of the IEEE, vol. 105, no. 4, pp. 756-767, 2017.

[32] W. Otter, N. Ridler, H. Yasukochi et al., "3D printed $1.1 \mathrm{THz}$ waveguides," Electronics Letters, vol. 53, no. 7, pp. 471-473, 2017.

[33] A. Gomez-Torrent, F. Teberio, A. Martinez et al., "A study of the additive manufacturing technology for $\mathrm{RF} /$ microwave components," in Proceedings of the 2017 11th European Conference on Antennas and Propagation (EUCAP), pp. 567-571, Paris, France, March 2017.

[34] H. Song, "Packages for Terahertz Electronics," Proceedings of the IEEE, vol. 105, no. 6, pp. 1121-1138, 2017.

[35] S. Khan, N. Vahabisani, and M. Daneshmand, "A fully 3-D printed waveguide and its application as microfluidically controlled waveguide switch," IEEE Transactions on Components, Packaging and Manufacturing Technology, vol. 7, no. 1, pp. 7080, 2017.

[36] E. A. Rojas-Nastrucci, J. Nussbaum, T. M. Weller, and N. B. Crane, "Metallic 3D printed Ka-band pyramidal horn using binder jetting," in Proceedings of the 2016 IEEE MTT-S Latin America Microwave Conference (LAMC), pp. 1-3, Puerto Vallarta, Mexico, December 2016.

[37] E. A. Rojas-Nastrucci, H. Tsang, P. I. Deffenbaugh et al., "Characterization and modeling of K-band coplanar waveguides digitally manufactured using pulsed picosecond laser machining of thick-film conductive paste," IEEE Transactions on Microwave Theory and Techniques, no. 99, pp. 1-8, 2017.

[38] A. I. Dimitriadis, T. Debogovic, M. Favre et al., "Polymer-based additive manufacturing of high-performance waveguide and antenna components," Proceedings of the IEEE, vol. 105, no. 4, pp. 668-676, 2017.

[39] K. Van Caekenberghe, P. Bleys, T. Craeghs, M. Pelk, and S. V. Bael, "A W-band waveguide fabricated using selective laser melting," Microwave and Optical Technology Letters, vol. 54, no. 11, pp. 2572-2575, 2012.

[40] B. Zhang, Y. X. Guo, H. Zirath, and Y. P. Zhang, "Investigation on 3-D-printing technologies for millimeter-wave and $\mathrm{THz}$ applications," in Proceedings of the IEEE, vol. 105, pp. 723-736, 4 edition, 2017.

[41] B. Zhang, P. Linnér, C. Kärnfelt, P. L. Tarn, U. Södervall, and H. Zirath, "Attempt of the metallic 3D printing technology for millimeter-wave antenna implementations," in Proceedings of the Asia-Pacific Microwave Conference (APMC '15), pp. 1-3, Nanjing, China, December 2015.

[42] B. Zhang, Z. Zhan, Y. Cao et al., "Metallic 3-D printed antennas for millimeter- and submillimeter wave applications," IEEE Transactions on Terahertz Science and Technology, vol. 6, no. 4, pp. 592-600, 2016. 
[43] B. Zhang and H. Zirath, "Metallic 3-D printed rectangular waveguides for millimeter-wave applications," IEEE Transactions on Components, Packaging and Manufacturing Technology, vol. 6, no. 5, pp. 796-804, 2016.

[44] B. Zhang and H. Zirath, "3D printed iris band-pass filters for millimeter-wave applications," Electronics Letters, vol. 15, no. 22, pp. 1791-1893, 2015.

[45] B. Zhang and H. Zirath, "A metallic 3-D printed E-band radio front end," IEEE Microwave and Wireless Components Letters, vol. 26, no. 5, pp. 331-333, 2016. 


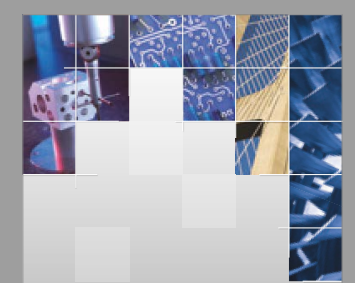

\section{Enfincering}
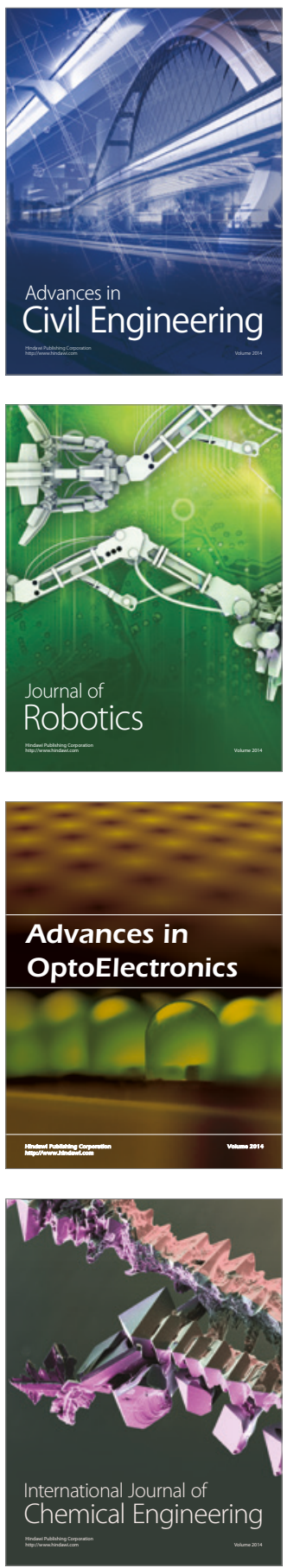

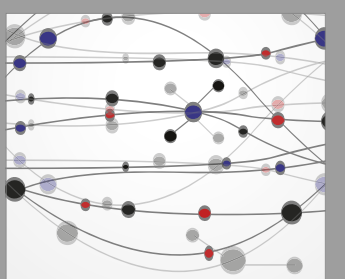

The Scientific World Journal

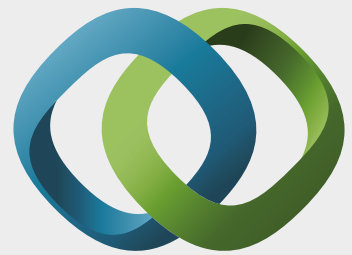

\section{Hindawi}

Submit your manuscripts at

https://www.hindawi.com
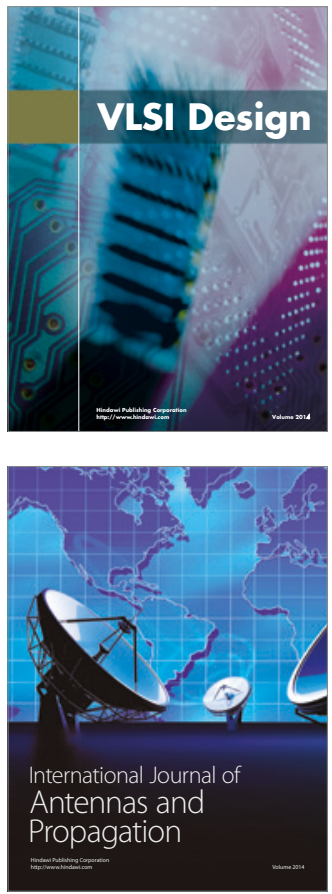

\section{Rotating}

Machinery
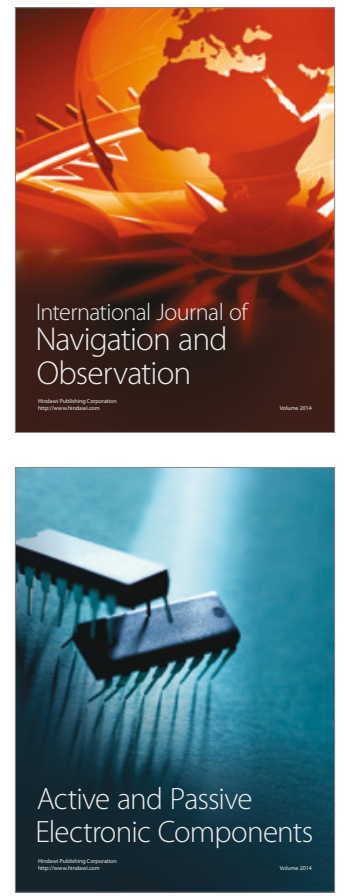
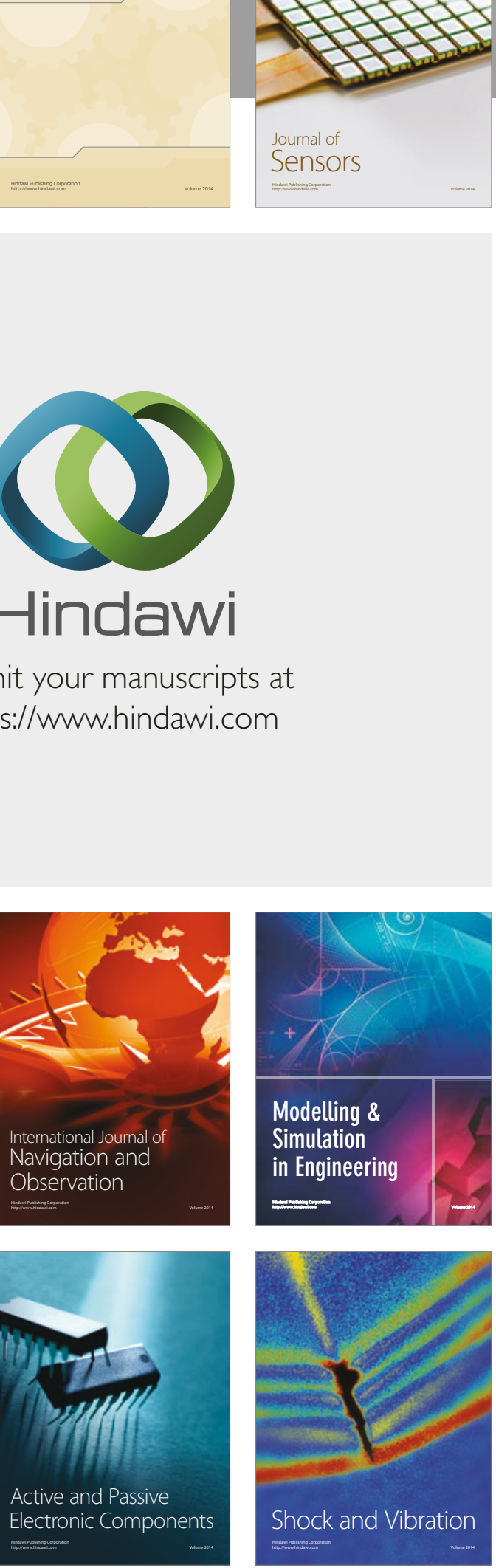
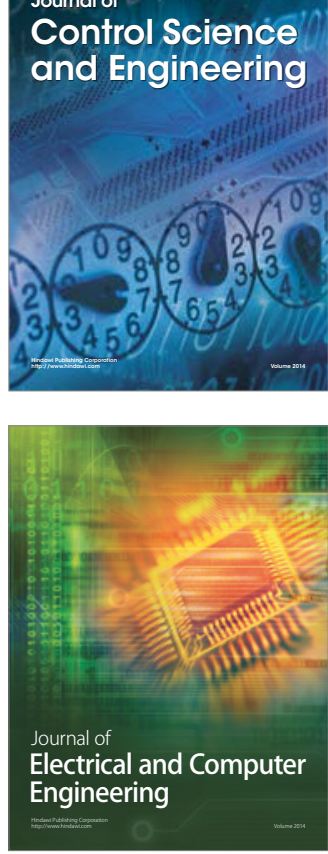

Distributed

Journal of

Control Science

and Engineering
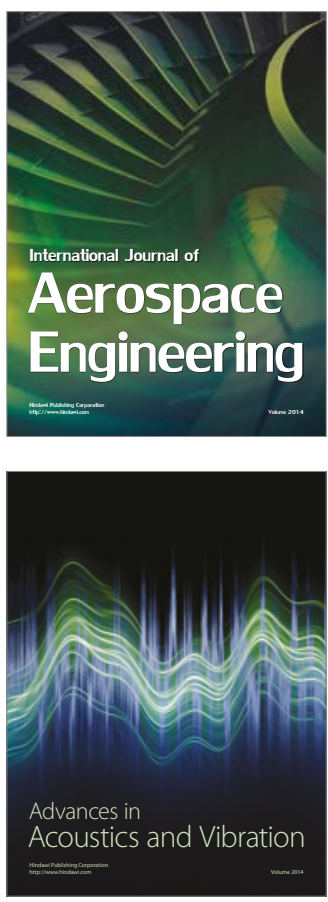

Sensor Networks 